\title{
Twitter Sentiment at the Hospital and Patient Level as a Measure of Pediatric Patient Experience
}

\author{
Samantha Robinson ${ }^{1 *}$, Ellie Vicha ${ }^{2}$ \\ ${ }^{1}$ Department of Mathematical Sciences, University of Arkansas, Fayetteville, USA \\ ${ }^{2}$ Department of Biological Sciences, University of Arkansas, Fayetteville, USA \\ Email: *sewrob@uark.edu
}

How to cite this paper: Robinson, S. and Vicha, E. (2021) Twitter Sentiment at the Hospital and Patient Level as a Measure of Pediatric Patient Experience. Open Journal of Pediatrics, 11, 706-722. https://doi.org/10.4236/ojped.2021.114066

Received: October 29, 2021

Accepted: December 5, 2021

Published: December 8, 2021

Copyright () 2021 by author(s) and Scientific Research Publishing Inc. This work is licensed under the Creative Commons Attribution International License (CC BY 4.0).

http://creativecommons.org/licenses/by/4.0/ (c) (i) Open Access

\begin{abstract}
Although pediatric cancer survival rates have improved, cancer is still the leading cause of death by disease among children in the United States. With nearly 16,000 new diagnoses each year of children having an average age of six years old, there is still much to be done to improve survival rates among children diagnosed with cancer. Additionally, clinical outcomes can be greatly improved by first understanding the patient perspective and, consequently, there is a need to understand the pediatric cancer patient experience. Interest in the analysis of patient-reported outcome (PRO) measures, including those specific to patient experience, has grown in recent years as attempts are made to understand and assess latent (i.e., unobserved) traits such as quality of life. While most traditional PRO measures involve lengthy and costly surveys that are limited in scope, posts on social media platforms are frequently lauded as more authentic, unbiased measures of patient experience. While many previous studies have utilized Twitter as a source for patient experience data that is not easily captured by traditional PROs, very few studies have examined the use of Twitter data to explore and better understand the experience of pediatric cancer patients specifically. Lexicon-based sentiment analysis of Twitter data was used both to examine the pediatric cancer patient experience as well as to compare the attitudes, perceptions, and overall impressions of individuals with St. Jude experiences to those with more general experiences. Results indicated that patients reflected on their care with more relative negativity when speaking of experiences other than St. Jude. While there were some notable differences between the two comparison groups, the sentiment of both was still mostly positive. Pediatric oncology differs greatly from adult oncological care and, thus, the proposed use of sentiment analysis with patient social media posts serving as authentic PRO data likely differentiates
\end{abstract}


more between medical providers and clinical care settings when assessing adult patient experience. Despite limitations, this exploratory study suggests that Twitter can be utilized as a source for authentic, real-time patient experience data that will improve overall care and better clinical outcomes, even among the pediatric cancer patient population.

\section{Keywords}

Twitter, Health Data Acquisition, Patient Experience, Self-Report Measures, Cancer

\section{Introduction}

While overall cancer death rates have decreased an average of $1.5 \%$ per year from 2013 to 2017 with notable decreases observed in common cancers such as lung and colorectal, the most recent Annual Report to the Nation on the Status of Cancer reveals that cancer incidence rates among children increased during this same time period [1]. As the number of children experiencing cancer as well as the expected survival times for those children increase, it is imperative to understand the pediatric cancer patient experience. Healthcare decisions and actions that are informed by the patient's point of view are critical to improving the value of that healthcare; knowledge of the patient experience can result in better treatments, symptom management, and long-term clinical outcomes. Patient-Reported Outcome (PRO) measures quantify patient experience and perspective, assessing how patients feel and function while receiving care. However, static PRO measures are time-consuming and collect very limited information about the entirety of the patient experience, oftentimes leading to inadequate buy-in from patients and clinicians. Previous studies have shown that unstructured, free-text information on social media platforms may reflect attitudes, beliefs, and experiences uncaptured with traditional surveys. The purpose of this study was to explore the utility of Twitter as a source of more authentic PRO data for pediatric cancer patients and to understand the experience of these patients both at the most well-known pediatric treatment and research facilities (i.e., St. Jude Children's Research Hospital) and overall.

\section{Literature Review}

\subsection{Pediatric Cancer}

According to Children's Miracle Network, one out of every 285 children in the United States will receive a cancer diagnosis before their twentieth birthday [2]. This equates to roughly 15,000 children diagnosed every year. In 2020, it is predicted that 1190 of these children will lose their lives to the disease [3]. While survival rates have improved greatly-from $58 \%$ in the 1970 s to $84 \%$ now-research focused on this specialized patient population, specifically their experiences with 
care, is still lacking and much is unknown.

Pediatric cancer comes in twelve main forms with over 100 subtypes [2]. Typically for these types of cancers, a child's risk of developing cancer is independent of lifestyle choices or personal behaviors. Even genetic abnormalities cause only a small percentage of pediatric cancer. Consequently, prevention-based research is less needed than research focused on treatment efficacy, treatment development, and the effects (both short- and long-term) of treatment and of the disease itself.

While cancer may appear to be similar in children and adults, pediatric cancers vary greatly. With better odds of remission but a greater risk of lasting impact, these patients require special treatment [4]. In a study looking at the survival rates of pediatric and adult cancer patients, results indicated that pediatric cancer patients fare better when treated at Children's Oncology Groups compared to when treated at Non-Children's Oncology Groups while the reverse is true of adults [5]. Pediatric and adult cancer must be treated as distinct diseases, just as pediatric and adult cancer patients must be treated as having distinct experiences in treatment. Children and adults will process cancer diagnoses and subsequent treatments in vastly different ways. Research related to the psycho-oncology of cancer patients is lacking in general, more so research differentiating the psycho-oncology of children and adult patients.

Pediatric cancer impacts a child for life yet research that takes a holistic approach to these specific patients, focusing on their wellness and not just their illness, is currently limited. While improving treatments for childhood cancer is extremely important, research must also focus on the child as a person and, consequently, explore the physical and mental effects of pediatric cancer. Research must explore the overall experience of the pediatric cancer patient in treatment, remission, and long-term.

\subsection{Measurement of Patient Experience}

According to the Patient-Centered Outcomes Research Institute [6], patient experience and patient perspective is essential to fully assess the clinical effectiveness and overall benefit of a treatment. Outcomes of critical importance to the population of interest, which are both patient-centered and relevant to decision makers e.g., health-related quality of life (HRQL), should be included in studies [6]. Oftentimes, patients are the best source of information to measure such outcomes and, consequently, PRO measures are recommended by and included in the PCORI methodology standards [6].

The U.S. Food and Drug Administration [7] defines a PRO measure as "any report of the status of a patient's health condition that comes directly from the patient, without interpretation of the patient's response by a clinician or anyone else." PROs are typically survey instruments and questionnaires designed to measure and monitor, among other things, patient-reported HRQL, symptoms, symptom frequency and severity, functional status, satisfaction with care, and 
treatment adherence [8] [9] [10].

According to Wilson and Cleary [11], the benefit of PRO data is that they can measure what the patient experiences during their care or course of treatment, thereby complementing traditional biological and physiological outcome data to provide a fuller picture of the relative efficacy of any particular treatment. Consequently, PROs are increasingly utilized in randomized controlled trials (RCTs) and clinical studies-for treatment evaluation, comparative effectiveness research (CER), drug development, and even population health monitoring [8] [12] [13] [14] [15] [16].

Using PRO data to supplement biological and physiological outcome data is especially important in cancer research, where survival-based end points are often not of sole concern to patients and their clinicians. Due to the increased survival times seen among cancer patients in recent decades, treatment choice must now balance efficacy and toxicity [17]. Patient experience is fundamental to determining the tolerability of cancer treatments. As cited in Kim et al. [18], the FDA Oncology Center of Excellence is committed to patient-focused drug development (PFDD) initiatives, which rely heavily on PRO data. As such, PRO measures are now fairly common in cancer trials, used predominantly to assess HRQL, physical functioning, and disease symptoms in patients. Research suggests the this use of PRO data improves cancer patient care as well as clinical outcomes such as treatment tolerance [19].

As cited in Nipp and Temel [19], Di Maio et al. found a lack of agreement in clinician and patient assessment of cancer symptom severity. Given the high symptom burden in cancer patients and the apparent information gap between patients and their clinicians as it relates to patient experience, it is particularly important to incorporate PRO measures into cancer research.

Cancer is quite varied though. As noted previously, pediatric cancer alone comes in twelve main forms with over 100 subtypes. With a wide variety of cancers and the development of several novel drug classes to treat these cancers, Kim et al. [18] argues that a "one-size-fits-all" approach to static PRO measures for HRQL in cancer studies is problematic. Static HRQL instruments do not necessarily capture symptomatic adverse events (AEs) that are common for cancer patients e.g., nausea, vomiting, and fatigue. Measures such as the $\mathrm{Pa}$ tient-Reported Outcomes version of the Common Terminology Criteria for Adverse Events (PRO-CTCAE) have been recently developed and identified by the FDA as a promising tool to expand the collection of meaningful and clinically relevant patient-reported data specific to the cancer patient population [18].

As noted by the new development of PRO measures explicitly targeting the cancer patient population, PROs are a benefit to the care of patients overall and for cancer patients in particular. Fortunately, the survival time for many cancer patients has increased; with this potential for a longer life however, the effects of the disease and its treatment are ever more important to measure, to quantify, and to research. PRO measures, by incorporating the patient's point of view and 
experiences, further patient-centered care, expand the care patients receive, and ultimately do so while improving clinical outcomes.

\subsection{Patient Experience Expressed through Social Media}

As recommended by PCORI [6] and the FDA [7], clinical trials including those specific to cancer research studies now routinely collect PRO data in some form. Clinical trials and, consequently, PRO data collected during those clinical trials lacks generalizability to the larger patient population, however. As noted by Mitchell et al. [20], participants in clinical trials are highly selective, unlikely to reflect the demographics of real-world patients. In fact, a literature review on the representativeness of RCT samples conducted by Kennedy-Marten et al. [21] revealed that over $70 \%$ of the studies reviewed explicitly concluded that RCT samples were not broadly representative of real-world patients. Especially in cancer related clinical trials, participants tend to be younger and have better diseases prognosis than real-world cancer patients [21]. Moreover, it is improbable that the settings of care involved in most clinical trials mirror those experienced by the larger patient population. Consequently, there is a need for PRO data that explores cancer patient experience, especially pediatric cancer patient experience, with greater external validity.

More authentic generalizable PRO data is needed to augment that collected during clinical trials. Moreover, PRO measures are needed that go beyond basic symptomatology and HRQL. A systematic review of research specific to cancer patients found that PRO data insufficiently captured the cancer patient experience and predominantly focused on symptoms reporting and patient tolerance to treatment [22]. Given the extensive impact cancer has on a patient's life, the review concluded that additional development of PRO measures is needed for cancer patients specifically. Additional measures that better capture the financial, employment, and parenting difficulties experienced by cancer patients could be used to better support and care for these patients.

Existing PRO measures also suffer from a lack of active participation from both patients and clinician. Response rates to traditional survey instruments, compliance with survey completion, participant attrition, and loss to follow-up all impact the validity of current PRO data. Basch et al. [23] found that offering cancer patients an electronic system for self-reporting purposes (i.e., an electronic $\mathrm{PRO}, \mathrm{ePRO}$ ) improved patient care. Incorporating ePROs mitigates to some degree the response and compliance issues of traditional PROs.

While integrating electronic systems is beneficial for PRO data collection, the measures themselves are still limited in scope. Current PROs, even if delivered electronically, are commonly survey instruments. Greaves et al. [24] noted that these survey instruments are expensive to administer yet provide inadequate information about the patient experience. The instruments themselves have a narrow set of prespecified questions with restrictive response choices. Moreover, these surveys are conducted infrequently at fixed time intervals and, conse- 
quently, lack the ability to capture the evolving patient experience in real time.

Outside of the structured PRO measures, patients already provide self-report of their experiences in real time on the Internet, especially on social media platforms (e.g., Facebook and Twitter) and health care websites (e.g., WebMD and PatientsLikeMe). Internet-based self-reports are not limited by survey instrument design or constrained to set timepoints. Patient experience is shared conveniently and with the additional benefit to the patient of social interactions [25]. The posted experiences often include clinically relevant information that is difficult to capture through conventional means of self-report. Posts made on social media platforms like Twitter are shared in real-time and provide a seemingly less biased snapshot of the patient experience than traditional surveys [26]. As proposed by Greaves et al. [24], the unstructured free-text data available from such online posts and comments, when analyzed with sentiment analysis, can serve as a real-time PRO measure that is either an alternative or a supplement to more traditional PROs.

Twitter is widely used in the United States and Mayol and Otero [27] report that Twitter is the most widely used social media platform even for healthcare professionals. Several recent studies have utilized Twitter data to study healthcare and the patient experience. In 2018, Sewalk and colleagues utilized sentiment analysis of Twitter data to examine differences in general patient experience across space and time in the United States [28]. Twitter data was also used to reveal differences in the sentiment of cancer patients and healthcare professionals regarding brachytherapy radiation treatment [29]. Sentiment analysis of Twitter data, thus, has already been used to identify patient needs and to measure patient experience, specifically in cancer patients [30].

While the number of studies examining cancer patient experiences using self-report data from social media platforms has increased and while the number of children diagnosed with cancer has also increased, there are still limited investigations using social media to better capture the pediatric cancer patient experience.

\subsection{Measurement of Pediatric Patient Experience}

As previously stated, despite the increase in the amount of pediatric cancer research, it is still nowhere near adequate and primarily focuses on treatment rather than the lived experiences of the patient [31]. Studies that focus on pediatric cancer research beyond the efficacy of treatment, have noted that complications of these treatments can result in late effects years later and that pediatric cancer survivors are of great interest since they make up a growing population [32]. Consequently, cancer survivorship research has recently emerged to better understand the long-term effects and impact of cancer among survivors.

Demonstrating the interest in cancer survivorship research among pediatric cancer patients, a multi-institutional, long term, follow up study funded by the National Cancer Institute of the National Institutes of Health i.e., the Childhood 
Cancer Survivor Study (CCSS) began in 1994 to explore the effect of pediatric cancer and its treatment on later health [33]. The CCSS is now composed of more than 30,000 survivors of pediatric cancers as well as over 5000 of their siblings. While the aim of the CCSS was to explore the long-term effects experienced by pediatric cancer survivors including behavioral and sociodemographic effects, research focused on the physical health effects of surviving pediatric cancer (e.g., second cancers, organ dysfunction, decreased fertility, etc.) are more prevalent than research focused on the psychosocial effects secondary to cancer (e.g., depression, anxiety, low self-esteem, etc.).

Among the studies that explore the psychosocial late effects in cancer survivors, early studies indicated that psychosocial sequelae are a function of age of onset and developmental disruption [34]. A later systematic review of psychosocial late effects in pediatric cancer survivors indicates that, while most survivors experience very few long-term problems, a subset experience increased rates of suicide ideation, global distress, anxiety, and post-traumatic stress disorder (PTSD) or similar symptoms [35]. This systematic review confirmed that certain demographics continued to be risk factors, highly correlated with psychosocial functioning, such as the age of disease onset. Bitsko and colleagues [35] also recommend that survivors' health beliefs and perceptions should be utilized over and above clinician ratings as predictors of suicide ideation, anxiety, and other psychosocial conditions.

Although the perception of pediatric cancer survivors is imperative for assessing and surveying late psychosocial effects, understanding the perception and perspective of current pediatric cancer patients is imperative for advancing patient care and improving clinical outcomes in real time while potentially preventing psychosocial late effects in pediatric cancer survivors. Receiving therapy throughout treatment has proven to be beneficial for children, as it allows for an open dialogue [36]. While receiving therapy is shown to be beneficial, the psychological trauma of pediatric cancer strongly alters the overall experience of the child [37]. Research is limited that focuses solely on the child's psychological condition and experiences throughout pediatric cancer, not in survival.

Cancer research with pediatric patients has many more complications than similar research with adult patients, as children may be too young to voice their experiences. Additionally, conventionally collected patient-report data may, in fact, have to come from parents or other caregivers. Nonetheless, pediatric cancer patient experience data is important, as it can result in a better environment of care, an improved treatment plan, and superior long-term outcomes while still allowing kids to be kids. To achieve a holistic view of the child and to adequately gauge the pediatric cancer patient experience, it is necessary to follow the evolving feelings and emotions of the child from diagnosis to remission in real time, learning from every stage of the child's journey.

The lived experiences of pediatric cancer patients, throughout their entire cancer journey and in real time, are rarely studied. Self-report of this lived expe- 
rience is one of the most accurate means to obtain a widespread and comprehensive understanding of the pediatric patient experience. While many studies have utilized Twitter as a source for real time and seemingly unbiased PRO data among adult cancer patients, few if any studies have examined the use of Twitter data to explore and better understand the experience of pediatric cancer patients. The current exploratory study focuses specifically on this patient population, exploring the utility of Twitter as a source of PRO data for pediatric cancer patients both at St. Jude and overall.

\section{Methods}

\subsection{Purpose of the Study \& Data Collection}

Twitter, with its amassed collection of self-reported, unstructured, free-text data, potentially reflects experiences uncaptured by traditional PRO data. This study aimed to gain further understanding of the pediatric patient experience, focusing on the experiences of patients at St. Jude and elsewhere in the United States.

In order to do so, Twitter data was obtained through one of the company's standard Application Programming Interfaces (APIs). Twitter was queried and data extracted in December 2019, resulting in a random sample of 3000 tweets related to the search term "St. Jude" and a second random sample of 3000 tweets containing the hashtag \#ChildhoodCancer.

Both samples of social media posts were restricted by ISO 639-1 language code and geocode such that all Tweets were in the English language and originated within a 1950 mile radius of the geographic center of the 48 contiguous United States, which restricted data collection to the North American continent. Figure 1

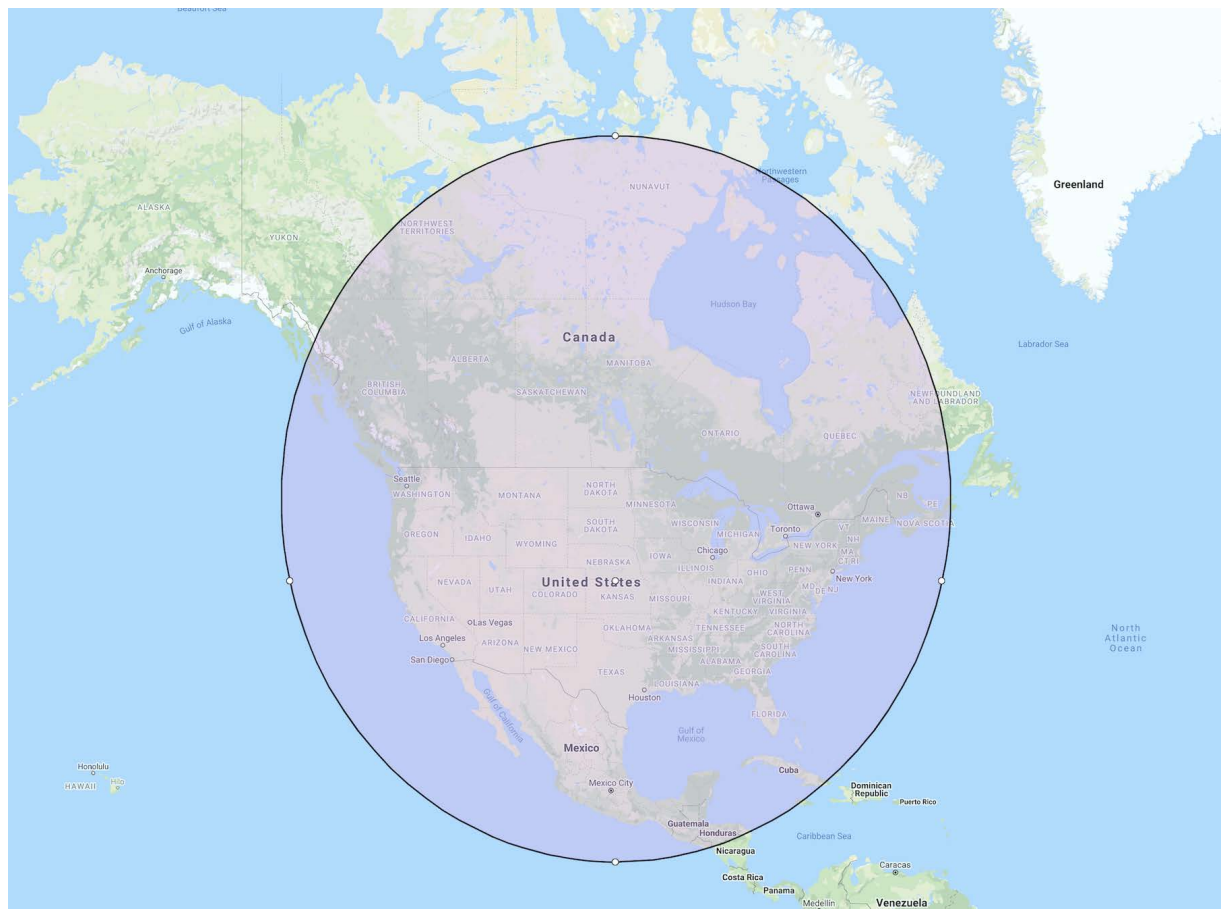

Figure 1. Geocode restriction for data collection. 
allows for a visualization of the geocode restriction.

The search term of "St. Jude" was used to extract social media posts that would serve as a proxy to explore the pediatric cancer patient experience at St. Jude, while the hashtag \#ChildhoodCancer was used to explore the pediatric cancer patient experience overall. The hashtag of \#ChildhoodCancer was selected because, at the time of analysis, hashtag reports indicated it was the most popular and frequently occurring hashtag related to pediatric cancer. As such, extracting social media posts utilizing this hashtag would result in a larger sample and was also hypothesized to be more encompassing of the overall pediatric cancer patient than more specific hashtags.

In order to have samples of the same initial size (prior to data pre-processing), Tweets in each of the two samples spanned different lengths of time. While extracted from Twitter on the same day, differences in posting frequencies of the two searches resulted in samples of social media posts that covered overlapping but differing time windows. For instance, Tweets associated with St. Jude spanned approximately forty days prior while Tweets with the hashtag \#ChildhoodCancer were posted during a period of approximately eleven days prior to Twitter query and data extraction.

All ethical standards of data collection were adhered to during this study. The data was publicly available and accessible through a Twitter API and, as such, are typically considered nonhuman participant research. However, in an effort to further protect the individuals creating and therefor indirectly supplying the extracted Twitter data, no identifiable Twitter information (including usernames, direct quotations, etc.) was reported.

\subsection{Analytic Approach}

All data pre-processing and analyses were performed in $\mathrm{R}$ [38].

Lexicon-based sentiment analysis was used to both explore and to compare the attitudes, perceptions, and overall impressions of individuals with St. Jude experiences to those individuals with more general childhood cancer experiences.

Prior to analysis, several data pre-processing steps were taken, specifically, word removal, word replacement, lowercasing, and punctuation removal. Each individual tweet was turned into a string of lowercase letters and punctuation such as periods, exclamation marks, and commas was removed. Manual word replacement was performed to replace misspelled words with their correctly spelled counterparts as well as to replace synonymous words with the most frequently used version of the word. Word removal took three forms: 1) stop words e.g., "and", "the", "a", etc. were removed; 2) other words with less than three characters were removed, as these were believed to be potential stop words or nonsensical exclamations not previously removed; 3) study-specific undesirable words were removed if it was determined that these words would not provide additional information in the study context e.g., "cancer", "St Jude", etc. Additionally, to limit redundancy and to attempt to capture only self-report, indivi- 
dually-created text data, all re-tweets (i.e., content that is shared by an individual but was originally authored by another) were removed.

Following data pre-processing, three different general-purpose lexicons available through the tidytext package were considered:

1) Bing [39];

2) National Research Council, NRC [40];

3) AFINN [41].

The National Research Council (NRC) lexicon contains a large number of words and, consequently, the match ratio of words common between the tweets and the lexicon was expected to be larger than that of a smaller lexicon such as AFINN. Moreover, the NRC lexicon, unlike the other two, assigns words to ten different categories of sentiment (i.e., anger, anticipation, disgust, fear, joy, negative, positive, sadness, surprise, and trust). Given the exploratory nature of the current study and the desire to more completely capture the pediatric cancer experience in sentiment, the ability of the NRC lexicon to classify sentiment beyond simply negative, neutral, or positive was seen to be beneficial. As such, the NRC lexicon was utilized for sentiment analysis.

\section{Results}

\subsection{Sentiment Analysis Results for St. Jude Children's Hospital}

NRC lexicon-based sentiment analysis was performed in $\mathrm{R}$ using pre-processed Twitter data from 3000 extracted tweets related to St. Jude in order to explore the pediatric cancer patient experience at one of the nation's most recognized pediatric treatment and research facilities. St. Jude Children's Research Hospital has a specific focus on childhood diseases, especially cancers, and is renowned for the fact that no patient is charged for care at the facility.

Figure 2 displays the overall distribution of Twitter sentiment scores for St. Jude. Tweets were assigned mostly positive sentiment scores, especially relative to negative sentiment scores within the sample. Anticipation, joy, and trust sentiments were also repeatedly observed.

Individual word frequencies were examined. Some of the most frequently observed words for the sample of St. Jude tweets were: "thanks", "danny thomas", "support", "treatment", "learn", and "awareness". These word frequencies reveal the thankfulness of care for those posting on Twitter, the importance of and appreciation for founder Danny Thomas to St. Jude, and the overall support felt at St. Jude.

Taken together, the sentiment scores and the examination of individual word frequency reveal an overall positive pediatric cancer patient experience at St. Jude Children's Research Hospital characterized by trust and support despite the anticipation inherent in the disease.

\subsection{Sentiment Analysis Results for \#ChildhoodCancer}

NRC lexicon-based sentiment analysis was also performed in $\mathrm{R}$ using pre-processed 


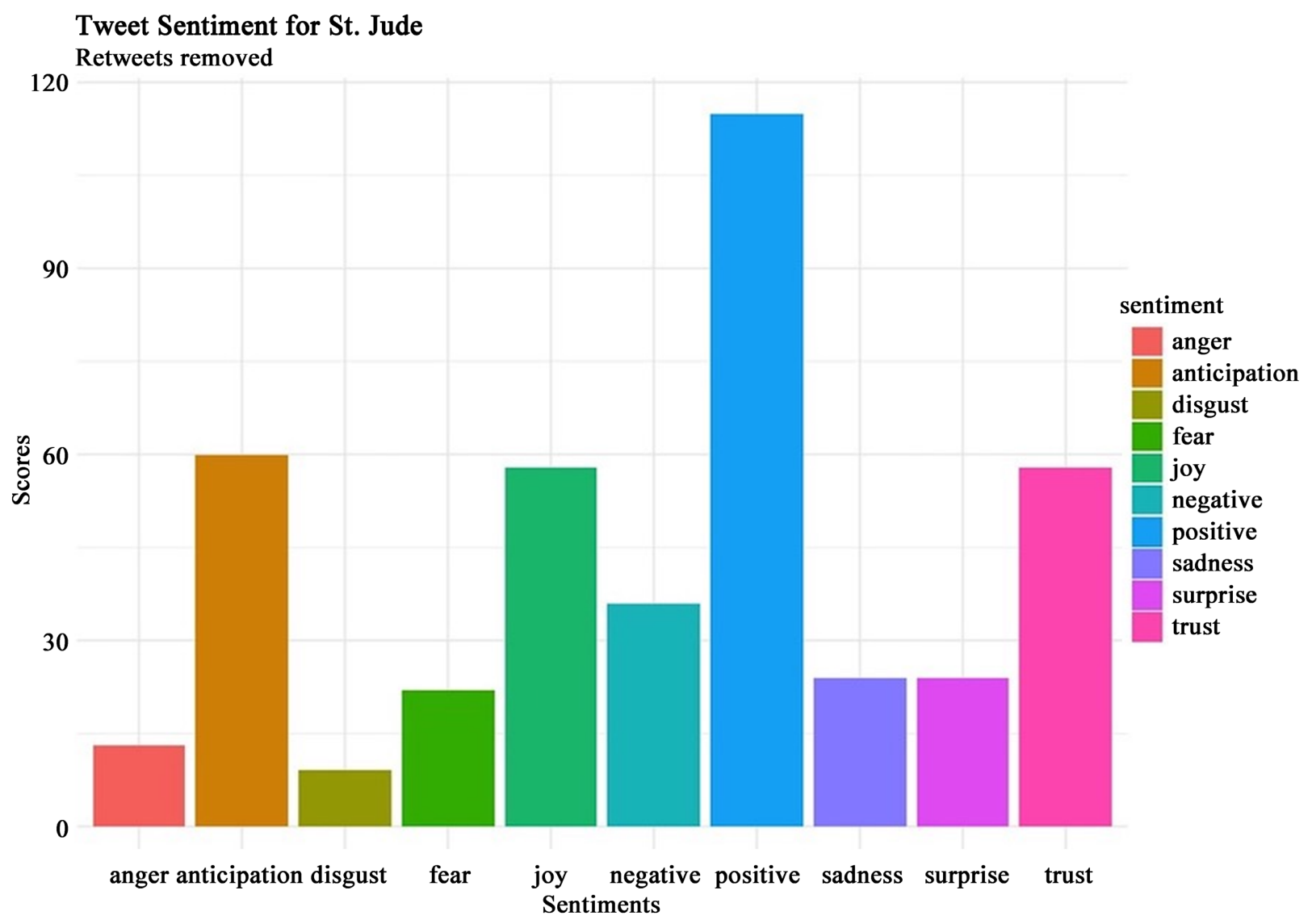

Figure 2. Distribution of Twitter Sentiment for St. Jude.

Twitter data from 3000 extracted tweets containing the hashtag \#ChildhoodCancer to explore the pediatric cancer patient experience overall, including that of patients receiving care in clinical settings other than St. Jude.

Figure 3 displays the overall distribution of Twitter sentiment scores for \#ChildhoodCancer. Tweets from this more general sample were also assigned mostly positive sentiment scores. Anticipation, joy, and negative sentiments were observed often and at approximately the same levels.

Individual word frequencies were examined. Some of the most frequently observed words for the sample of \#ChildhoodCancer tweets were: "love", "life", "inspirational", "remarkable", "cruel", "blow", "losing", "heartache", "abuse", and "back". These word frequencies reveal the cruel heartache, yet inspirational experiences associated with pediatric cancer patients, the benefit finding that can occur during the treatment process and cancer journey, and the confronting of failed treatment progress and disease recurrence.

\subsection{Comparison of Sentiment Results}

Comparison of the NRC lexicon-based sentiment analysis results for St. Jude and \#ChildhoodCancer, reveals a few interesting results. Results of a correlation analysis indicate that there is a significant and positive linear association between the NRC lexicon-based sentiment scores for each sample, $r(8)=0.97, \mathrm{p}<$ 


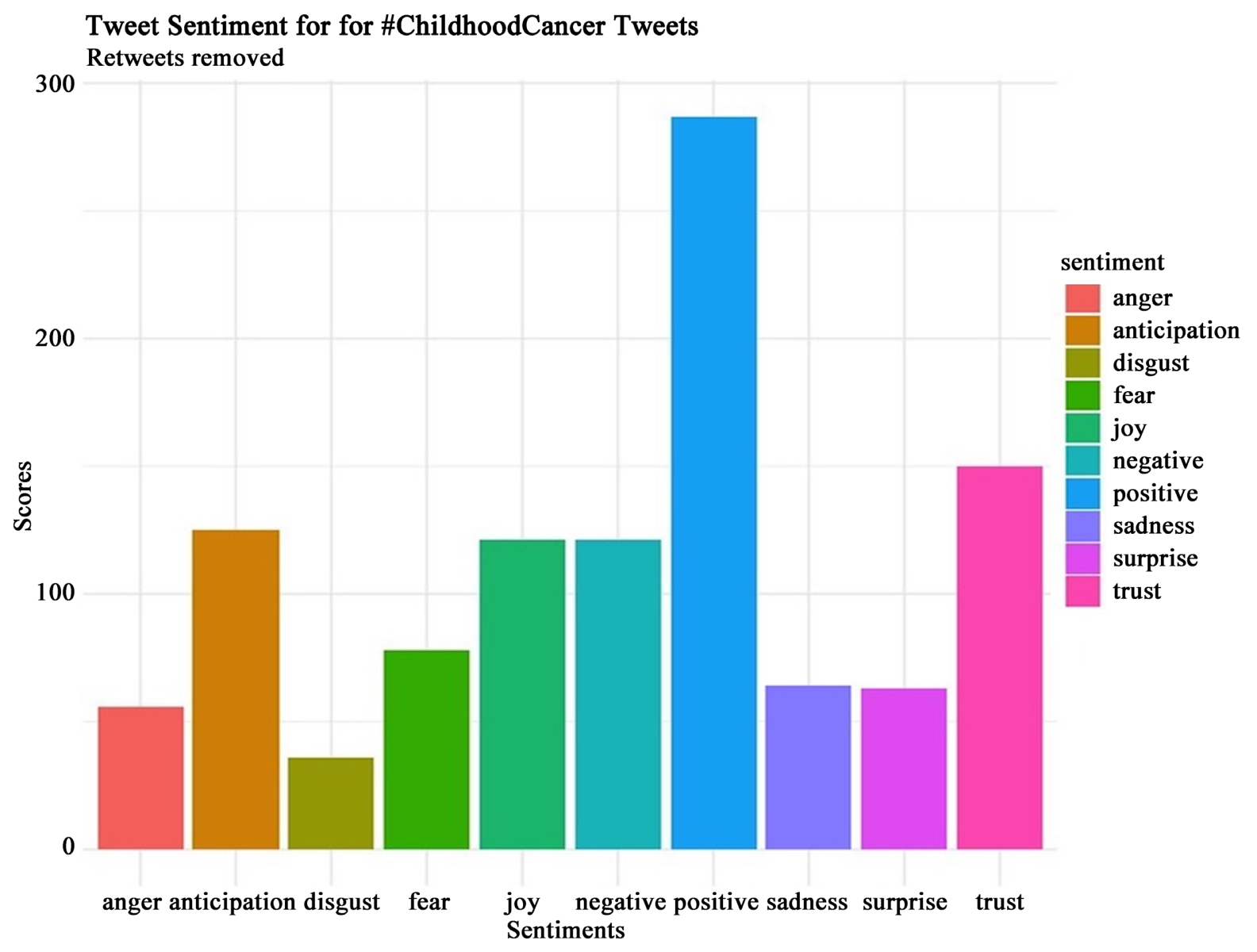

Figure 3. Distribution of Twitter Sentiment for \#ChildhoodCancer.

0.001. While overall sentiment scores indicate very little difference between the posts associated with St. Jude and those associated with the more general hashtag of \#ChildhoodCancer, there was greater relative negativity expressed generally than expressed for St. Jude when observing the positive-negative sentiment score ratio. The St. Jude pediatric cancer patient experience appears more relatively positive than in other settings of care.

Additionally, looking beyond the positive-negative sentiment score ratio, the distribution of sentiment scores for each sample further suggest that more relative negative sentiment was expressed in tweets containing the hashtag \#ChildhoodCancer than in tweets related to St. Jude. The St. Jude experience was approximately equally described as anticipation, joy, and trust. However, the more general experience was described as negative in nearly equivalent proportions as anticipation and joy.

Observed word frequencies indicated that, while there was a great deal of positivity associated with both comparison groups, words such as "cruel" emerged with much more frequency in general posts related to \#ChildhoodCancer than in posts related to St. Jude.

It appears that the pediatric cancer patient experience is both benefit and burden, both positive and negative, both inspirational and cruel. However, St. 
Jude appears to provide a more positive overall experience than other clinical care settings.

\section{Discussion}

Among cancer patients and, especially among pediatric cancer patients, the patient perspective is critical to ensuring desirable clinical outcomes during and after treatment. That pediatric cancer patient perspective can only come (in an unbiased manner) directly from the patient. When a concept of interest is best known by the patient, the FDA [7] recommends utilizing a PRO measure. However, conventional PRO measures are by nature limited in scope and leave various aspects of the patient experience uncaptured. Mirroring an approach taken in previous research to analyze more authentic and real-time PRO data but focusing specifically on the insufficiently studied pediatric cancer patient population, the current study utilized Twitter data to better understand the experience of pediatric cancer patients.

Results indicated that pediatric cancer patients report experiences that are relatively positive despite the physical and emotional burden of cancer diagnosis and treatment. This is in alignment with previous research that suggests pediatric cancer patients "report positive and negative aspects of their illness simultaneously" and engages in benefit finding similar to adult cancer patients [42]. Within the pediatric cancer community, optimism and positivity might be fairly standard. Consequently, self-reported experiences for one comparison group that is relatively more negative might be more predictive of treatment toxicity or settings of care that should be improved.

The results of this exploratory study demonstrate that Twitter has the potential to capture the pediatric cancer patient experience and that sentiment analysis can be a means to quantify, make comparisons, and better understand the experience. While Twitter data is a promising complement to traditional PRO data, there are limitations to this initial study and suggestions that can be made for future work.

While our goal was to analyze content authored only by pediatric cancer patients, it is not clear that this was accomplished. Just as conventional PRO measures for pediatric cancer patients often do not meet the formal definition of a PRO established by the FDA in 2009 because data is often reported with interpretation by parents; this could again be the case for Twitter-based PRO data. There is no means in the current study to definitively determine if Twitter data is being reported directly by the child patient or by a parent/guardian. In future work, pediatric cancer patient experience studies utilizing social media could focus exclusively on adolescent-aged patients, as these patients would be most capable and, thus, most likely to be posting for themselves without interpretation.

Additional limitations to Twitter data collection include keyword, hashtag, and user selection. In this current exploratory study of pediatric cancer patient 
experience, St. Jude related Twitter data and the hashtag of \#ChildhoodCancer were utilized for data queries. As noted by Kim et al. [43], the selection of terms used for data collection through the Twitter API often relies on a subjective decision by the researchers. Future studies should utilize additional hashtags, keywords, and/or users that are either targeted (i.e., specific to a particular cancer type) or perform several preliminary keyword searches to provide a more methodologically-guided approach to final keyword selection.

The current study, while similar to many previous studies, relied on only one single platform of social media (i.e., Twitter). Though this is now a fairly common social media platform for obtaining patient experience data, future work could incorporate self-report data that comes from other social media platforms (some of which might be more suitable and age-appropriate for the pediatric patient population).

Lexicon-based sentiment analysis also results in inherent limitations, as any one general-purpose lexicon may not be fully capable of adequately assigning sentiment scores in a more specific context [44]. If possible, future research could utilize a lexicon that is better able to capture sentiment in the realm of cancer treatment.

Future work could also explore sentiment over time for particular care settings, especially exploring the potential to detect structural change in sentiment following institutional interventions. Sentiment over time could also be examined at an individual patient level, following a pediatric cancer patient throughout the various stages of diagnosis, treatment, remission, and possible recurrence. Any analysis over time would be beneficial to better capture the pediatric cancer patient experience as it evolves.

While limitations exist in the current study and while further work is needed to fully assess and understand pediatric cancer patient experience, this study suggests that physicians and anyone involved in the care of pediatric cancer patients can use social media posts as a form of authentic, real-time patient-reported experience. Such information, serving as a supplement to traditional PRO measures, can then be used both to better understand the patient perspective and to better care for pediatric cancer patients.

\section{Conflicts of Interest}

The authors declare no conflicts of interest regarding the publication of this paper.

\section{References}

[1] Henley, S.J., Ward, E.M., Scott, S., Ma, J., Anderson, R.N., Firth, A.U., Thomas, C.C., Islami, F., et al. (2020) Annual Report to the Nation on the Status of Cancer, Part 1: National Cancer Statistics. Cancer, 126, 2225-2249. https://doi.org/10.1002/cncr.32802

[2] Children's Miracle Network (2020) Kicks for Kids. https://childrensmiraclenetworkhospitals.org/fundraiser/303 
[3] American Cancer Society (2020) Key Statistics for Childhood Cancers. https://www.cancer.org/cancer/cancer-in-children/key-statistics.html

[4] American Cancer Society (2019) Can Childhood Cancers Be Prevented? https://www.cancer.org/cancer/cancer-in-children/preventing-childhood-cancers.html

[5] Bleyer, A. (2010) The Quid Pro Quo of Pediatric versus Adult Services for Older Adolescent Cancer Patients. Pediatric Blood and Cancer, 54, 238-241. https://doi.org/10.1002/pbc.22190

[6] Patient-Centered Outcomes Research Institute (2019) PCORI Methodology Standards.

https://www.pcori.org/research-results/about-our-research/research-methodology/ pcori-methodology-standards

[7] U.S. Food and Drug Administration (FDA) (2009) Guidance for Industry Patient-Reported Outcome Measures: Use in Medical Product Development to Support Labeling Claims. http://www.fda.gov/downloads/Drugs/Guidances/UCM193282.pdf

[8] Calvert, M., Blazeby, J. and Altman, D.G. (2013) Reporting of Patient-Reported Outcomes in Randomized Trials: The CONSORT PRO Extension. JAMA, 309, 814-822. https://doi.org/10.1001/jama.2013.879

[9] Deshpande, P.R., Rajan, S., Sudeepthi, B.L. and Abdul Nazir, C.P. (2011) Patient-Reported Outcomes: A New Era in Clinical Research. Perspectives in Clinical Research, 2, 137-144. https://doi.org/10.4103/2229-3485.86879

[10] Fayers, P. and Machin, D. (2007) Quality of Life, the Assessment, Analysis and Interpretation of Patient-Reported Outcomes. 2nd Edition, Wiley, Chichester.

[11] Wilson, I. and Cleary, P. (1995) Linking Clinical Variables with Health-Related Quality of Life: A Conceptual Model of Patient Outcomes. JAMA, 273, 59-65. https://doi.org/10.4103/2229-3485.86879

[12] Ahmed, S., Berzon, R.A., Revicki, D.A., Lenderking, W.R., Moinpour, C.M., Basch, E., et al. (2012) The Use of Patient-Reported Outcomes (PRO) within Comparative Effectiveness Research: Implications for Clinical Practice and Health Care Policy. Medical Care, 50, 1060-1070. https://doi.org/10.1097/MLR.0b013e318268aaff

[13] Black, N. and Tan, S. (2013) Use of National Clinical Databases for Informing and for Evaluating Health Care Policies. Health Policy, 109, 131-136. https://doi.org/10.1016/j.healthpol.2012.10.007

[14] Gnanasakthy, A., Mordin, M., Clark, M., DeMuro, C., Fehnel, S. and Copley-Merriman, C. (2012) A Review of Patient-Reported Outcome Labels in the United States: 2006 to 2010. Value Health, 15, 437-442. https://doi.org/10.1016/j.jval.2011.11.032

[15] McGrail, K., Bryan, S. and Davis, J. (2011) Let's All Go to the PROM: The Case for Routine Patient-Reported Outcome Measurement in Canadian Healthcare. Healthcare Papers, 11, 8-18. https://doi.org/10.12927/hcpap.2012.22697

[16] Valderas, J.M., Kotzeva, A., Espallargues, M., Guyatt, G., Ferrans, C.E., Halyard, M.Y., Revicki, D.A., Symonds, T., Parada, A. and Alonso, J. (2008) The Impact of Measuring Patient-Reported Outcomes in Clinical Practice: A Systematic Review of the Literature. Quality of Life Research, 17, 179-193. https://doi.org/10.1007/s11136-007-9295-0

[17] Basch, E. (2013) Toward Patient-Centered Drug Development in Oncology. NEJM, 369, 397-400. https://doi.org/10.1056/NEJMp1114649

[18] Kim, J., Singh, H., Ayalew, K., Borror, K., Campbell, M., Johnson, L.L., Karesh, A., 
Khin, N.A., Less, J.R., Menikoff, J., Minasian, L., Mitchell, S.A., Papadopoulos, E.J., Piekarz, R.L., Prohaska, K.A., Thompson, S., Sridhara, R., Pazdur, R. and Kluetz, P.G. (2018) Use of PRO Measures to Inform Tolerability in Oncology Trials: Implications for Clinical Review, IND Safety Reporting, and Clinical Site Inspections. Clinical Cancer Research, 24, 1780-1784.

https://doi.org/10.1158/1078-0432.CCR-17-2555

[19] Nipp, R.R. and Temel, J.S. (2018) Harnessing the Power of Patient-Reported Outcomes in Oncology. Clinical Cancer Research, 24, 1777-1779.

https://doi.org/10.1158/1078-0432.CCR-18-0037

[20] Mitchell, A.P., Harrison, M.R., George, D.J., Abernethy, A.P., Walker, M.S. and Hirsch, B.R. (2014) Clinical Trial Subjects Compared to "Real World” Patients: Generalizability of Renal Cell Carcinoma Trials. Journal of Clinical Oncology, 32, 6510-6510. https://doi.org/10.1200/jco.2014.32.15_suppl.6510

[21] Kennedy-Martin, T., Curtis, S., Faries, D., Robinson, S. and Johnston, J. (2015) A Literature Review on the Representativeness of Randomized Controlled Trial Samples and Implications for the External Validity of Trial Results. Trials, 16, Article No. 495. https://doi.org/10.1186/s13063-015-1023-4

[22] Catt, S., Starkings, R., Shilling, V. and Fallowfield, L. (2017) Patient-Reported Outcome Measures of the Impact of Cancer on Patients' Everyday Lives: A Systematic Review. Journal of Cancer Survivorship, 11, 211-232.

https://doi.org/10.1007/s11764-016-0580-1

[23] Basch, E., Deal, A.M., Kris, M.G., Scher, H.I., Hudis, C.A., Sabbatini, P., Rogak, L., Bennett, A.V., Dueck, A.C., Atkinson, T.M., Chou, J.F., Dulko, D., Sit, L., Barz, A., Novotny, P., Fruscione, M., Sloan, J.A. and Schrag, D. (2019) Symptom Monitoring with Patient-Reported Outcomes during Routine Cancer Treatment: A Randomized Controlled Trial. Journal of Clinical Oncology, 34, 557-565.

https://doi.org/10.1200/JCO.2015.63.0830

[24] Greaves, F., Ramirez-Cano, D., Millett, C., Darzi, A. and Donaldson, L. (2013) Use of Sentiment Analysis for Capturing Patient Experience From Free-Text Comments Posted Online. Journal of Medical Internet Research, 15, e239.

https://doi.org/10.2196/jmir.2721

[25] Ru, B., Harris, K. and Yao, L. (2016) A Content Analysis of Patient-Reported Medication Outcomes on Social Media. Proceedings of the 15th IEEE International Conference on Data Mining Workshop, Barcelona, 12-15 December 2016, 472-479. https://doi.org/10.1109/ICDMW.2015.150

[26] Takabe, K. (2016) Twitter as a Survey Tool for Real-Time Unbiased Snapshots of Personal Sentiment in Population Level. Journal of Surgical Research, 206, 543-544. https://doi.org/10.1016/j.jss.2016.08.042

[27] Mayol, J. and Otero, J. (2017) Breaking International Barriers: \#ColorectalSurgery Is \#GlobalSurgery. Clinics in Colon and Rectal Surgery, 30, 277-280. https://doi.org/10.1055/s-0037-1604257

[28] Sewalk, K.C., Tuli, G., Hswen, Y., Brownstein, J.S. and Hawkins, J.B. (2018) Using Twitter to Examine Web-Based Patient Experience Sentiments in the United States: Longitudinal Study. Journal of Medical Internet Research, 20, e10043. https://doi.org/10.2196/10043

[29] Thomas, J., Prabhu, A.V., Heron, D.E. and Beriwal, S. (2018) Twitter and Brachytherapy: An Analysis of "Tweets" over Six Years by Patients and Health Care Professionals. Brachytherapy, 17, S36-S37. https://doi.org/10.1016/j.brachy.2018.07.015

[30] Crannell, W.C., Clark, E., Jones, C., James, T.A. and Moore, J. (2016) A Pat- 
tern-Matched Twitter Analysis of US Cancer-Patient Sentiments. The Journal of Surgical Research, 206, 536-542. https://doi.org/10.1016/j.jss.2016.06.050

[31] Berríos-Rivera, R., Rivero-Vergne, A. and Romero, I. (2008) The Pediatric Cancer Hospitalization Experience: Reality Co-Constructed. Journal of Pediatric Oncology Nursing, 25, 340-353. https://doi.org/10.1177/1043454208323618

[32] Bhatia, S. and Landier, W. (2005) Evaluating Survivors of Pediatric Cancer. The Cancer Journal, 11, 340-354. https://doi.org/10.1097/00130404-200507000-00010

[33] Robison, L.L., Armstrong, G.T., Boice, J.D., Chow, E.J., Davies, S.M., Donaldson, S.S., Green, D.M., Hammond, S., Meadows, A.T., Mertens, A.C., Mulvihill, J.J., Nathan, P.C., Neglia, J.P., Packer, R.J., Rajaraman, P., Sklar, C.A., Stovall, M., Strong, L.C., Yasui, Y. and Zeltzer, L.K. (2009) The Childhood Cancer Survivor Study: A National Cancer Institute-Supported Resource for Outcome and Intervention Research. Journal of Clinical Oncology. Official Journal of the American Society of Clinical Oncology, 27, 2390-2395. https://doi.org/10.1200/JCO.2009.22.3339

[34] Koocher, G.P., O’Malley, J.E., Gogan, J.L. and Foster, D.J. (1980) Psychological Adjustment among Pediatric Cancer Survivors. Journal of Child Psychology and Psychiatry, 21, 163-173. https://doi.org/10.1111/j.1469-7610.1980.tb00028.x

[35] Bitsko, M.J., Cohen, D., Dillon, R., Harvey, J., Krull, K. and Klosky, J.L. (2016) Psychosocial Late Effects in Pediatric Cancer Survivors: A Report from the Children's Oncology Group. Pediatric Blood Cancer, 63, 337-343.

https://doi.org/10.1002/pbc.25773

[36] Haase, J.E. and Rostad, M. (1994) Experiences of Completing Cancer Therapy: Children's Perspectives. Oncology Nursing Forum, 21, 1483-1492.

[37] Portteus, A., Ahmad, N., Tobey, D. and Leavey, P. (2006) The Prevalence and Use of Antidepressant Medication in Pediatric Cancer Patients. Journal of Child and Adolescent Psychopharmacology, 16, 46. https://doi.org/10.1089/cap.2006.16.467

[38] R Core Team (2019) R: A Language and Environment for Statistical Computing. R Foundation for Statistical Computing, Vienna. https://www.R-project.org

[39] Liu, B. (2012) Sentiment Analysis and Opinion Mining. Synthesis Lectures on Human Language Technologies, 5, 1-167. https://doi.org/10.2200/S00416ED1V01Y201204HLT016

[40] Mohammad, S.M. and Turney, P.D. (2013) Crowdsourcing a Word-Emotion Association Lexicon. Computational Intelligence, 29, 436-465. https://doi.org/10.1111/j.1467-8640.2012.00460.x

[41] Nielsen, F.Å. (2011) A New ANEW: Evaluation of a Word List for Sentiment Analysis in Microblogs. Proceedings of the ESWC2011 Workshop on "Making Sense of Microposts": Big Things Come in Small Packages, Vol. 718, 93-98.

[42] Currier, J.M., Hermes, S. and Phipps, S. (2009) Brief Report: Children's Response to Serious Illness: Perceptions of Benefit and Burden in a Pediatric Cancer Population. Journal of Pediatric Psychology, 34, 1129-1134. https://doi.org/10.1093/jpepsy/jsp021

[43] Kim, H., Jang, S.M., Kim, S.H. and Wan, A. (2018) Evaluating Sampling Methods for Content Analysis of Twitter Data. Social Media + Society, 4, 1-10. https://doi.org/10.1177/2056305118772836

[44] Khoo, C.S. and Johnkhan, S.B. (2018) Lexicon-Based Sentiment Analysis: Comparative Evaluation of Six Sentiment Lexicons. Journal of Information Science, 44, 491-511. https://doi.org/10.1177/0165551517703514 